\title{
Using Cucurbits By-Products as Reagents for Disposal of Pollutants from Water Environments (a Literature Review)
}

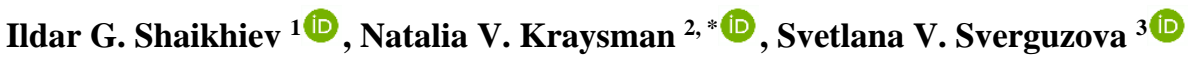 \\ 1 Department of Engineering Ecology, Institute of Chemical Engineering and Technology, Kazan National Research \\ Technological University, Kazan, Russian Federation; ildars @inbox.ru (I.G.S.); \\ 2 Department of Foreign Languages for Professional Communication, Institute of Innovation Management, Kazan National \\ Research Technological University, Kazan, Russian Federation; n_kraysman@mail.ru (N.V.K.); \\ 3 Department of Industrial Ecology, Institute of Chemical Engineering and Technology, Belgorod State Technological \\ University named after V.G. Shukhov, Belgorod, Russian Federation; pe@ intbel.ru (S.V.S.); \\ * Correspondence: n_kraysman@mail.ru;
}

Scopus Author ID 56114338900

Received: 16.12.2020; Revised: 16.01.2021; Accepted: 22.01.2021; Published: 31.01.2021

\begin{abstract}
The paper summarizes data from literature sources using the waste of processing cucurbits (pumpkin, watermelon, melon, cucumber) as a sorption material to remove various pollutants from water environments. It states that cucurbits' shells are effective sorption materials for extracting heavy metal ions and dyes. Seeds of large cucurbits fruits (pumpkin, watermelon, melon) and seed shells also showed good sorption performance for heavy metal ions and dyes. It was found that most of the pollutants adsorption isotherms on cucurbits by-products are most accurately described by the Langmuir model, less often by the Freundlich model, and occasionally by the Tyomkin or Dubinin-Radushkevich models. It was determined that the adsorption process kinetics most often follows the pseudo-secondorder model, less often - the logistic model. To increase the adsorption characteristics of cucurbits fruit components for various pollutants, they were modified with various chemical reagents.
\end{abstract}

Keywords: cucurbits; by-products; water treatment; adsorption; heavy metal ions; dyes; modification.

(C) 2021 by the authors. This article is an open-access article distributed under the terms and conditions of the Creative Commons Attribution (CC BY) license (https://creativecommons.org/licenses/by/4.0/).

\section{Introduction}

The world community is currently rapidly developing a new innovative environmental protection area - the use of industrial and agricultural waste as reagents for removing pollutants from water environments. By-products of agricultural raw materials are of particular importance. The latter are formed annually on a large scale, are renewable, very cheap, contain natural compounds and biopolymers with various functional groups, promoting their widespread use in various sectors of the national economy.

The world literature provides much information about the use of agricultural raw materials waste as coagulants and flocculants, sorption materials for various pollutants removal from water [1-14].

A fairly large role in human nutrition is played by cucurbits, usually those from the gourd family (Cucurbitaceae). The latter mainly include pumpkins, watermelons, melons, marrows, scallop squashes, cucumbers. When they are processed, various wastes are formed, usually the rind of fruits and seeds. 


\section{The Use of Pumpkin Fruit Waste as Sorption Materials for Removal of Metal Ions and Dyes from Aqueous Media}

The most important of these crops for people are pumpkins (Cucurbita), i.e., herbaceous plant genus in the gourd family (Cucurbitaceae). The word "pumpkin" usually refers to the common pumpkin (Cucurbita pepo) and winter squash (Cucurbita maxima), which are widely spread and cultivated as food and fodder plants. Annual or perennial rough or hairy grasses that creep along the ground and cling with branched tendrils. The stems are covered with more or less large lobed leaves. The fruit is a pumpkin, normally with a hard outer layer (skin) and numerous flattened seeds framed by thick bloat [15].

Pumpkin is a fruit that has a high nutritional value due to its high content of carbohydrates, soluble fiber, protein, carotenoid compounds, vitamin A precursors, $\beta$-carotene, cryptoxanthin, and lutein [16]. Epy seeds are flat, oval, of various colors, and are used for food and medicinal purposes. Pumpkin seeds are rich in oil and nutrients. In particular, the composition of pumpkin seeds was determined in [17]: humidity $-4.06 \%$, ash content $-3.80 \%$, fiber $-2.91 \%$, fat $-36.70 \%$, protein $-34.56 \%$, soluble protein $-18.1 \%$, sugar $-1.08 \%$, and starch $-2.15 \%$. Pumpkin seeds contain $48.92 \%$ of oils, of which $29.04 \%$ is oleic acid, $51.87 \%$ - linoleic acid, 11.64\% - palmitic acid, and 7.0\% - stearic acid [18].

Processing pumpkin fruits results in waste in the form of fruit rind and seeds. There are various ways to use the waste from processing cucurbits, including pumpkins, in the national economy [19]. The latter has also been studied as sorption materials for extracting various pollutants from natural and wastewater.

Muscat pumpkin (Cucurbita moschata Duchesne ex Poir) rind, dried and treated with $1 \mathrm{~mol} \mathrm{KOH}$ solution, was studied as a sorption material for extracting $\mathrm{Cu}^{2+}$ ions from simulated solutions. It was found that the maximum sorption capacity for these ions is $78.13 \mathrm{mg} / \mathrm{g}$ at an initial $\mathrm{Cu}^{2+}$ concentration of $80 \mathrm{mg} / \mathrm{dm}^{3}$, a temperature of $298 \mathrm{~K}, \mathrm{pH}=6.3$, and a dosage of the sorption material of $0.5 \mathrm{~g} / \mathrm{dm}^{3}$. It was determined that the adsorption isotherms at $298-318 \mathrm{~K}$ are most adequately described by the Freundlich equation, and the process kinetics is described by the logistic model [20].

Native and carboxylated common pumpkin rind was studied to extract $\mathrm{Cd}^{2+}, \mathrm{Hg}^{2+}, \mathrm{Pb}^{2+}$, and $\mathrm{Zn}^{2+}$ ions [21]. At the initial concentration of these ions of $100 \mathrm{mg} / \mathrm{dm}^{3}$, the dosage of the sorption material of $1.2 \mathrm{~g} / \mathrm{dm}^{3}, \mathrm{pH}=7.5$ and 1-hour contact, the degree of metal ions removal by native pumpkin rind was $67 \%, 52 \%, 85 \%$, and $78 \%$, respectively. For a carboxylated sample of pumpkin rind, these ions' removal rates were $73 \%, 60 \%, 65 \%$, and $95 \%$, respectively.

The dried and ground rind of muscat pumpkin was also studied to extract Lanaset Red G [22] and Reactive Red 120 [23] dyes from simulated solutions. It was found that the maximum sorption capacity for these dyes was 440.78 and $98.61 \mathrm{mg} / \mathrm{g}$, respectively. In both cases, the isotherms of dye adsorption in the temperature range of $298-328 \mathrm{~K}$ are most accurately described by the Freundlich equations. The logistic model describes the process kinetics. Determination of thermodynamic parameters showed that both cases' adsorption process is physical, exothermic, and spontaneous.

The adsorption of Acid Black 1 dye by dried native and modified by loading $\mathrm{Ni}^{2+}$ ions muscat pumpkin rind was studied. Modification with $\mathrm{Ni}^{2+}$ ions led to an increase in the rind sorption capacity towards the dye. It was determined that Acid Black 1 dye adsorption by these sorption materials increased with decreasing particle size, reagent dose, solution $\mathrm{pH}$, and temperature, but increased with increasing contact time and initial dye concentration. It was 
found that rapid adsorption occurred in the first 5 minutes, and kinetic equilibrium was reached within 50 minutes. The maximum sorption capacity towards the studied dye for native and $\mathrm{Ni}^{2+}$-modified pumpkin rind was $227.62 \mathrm{mg} / \mathrm{g}$ and $228.49 \mathrm{mg} / \mathrm{g}$, respectively. The Freundlich equation most accurately described the adsorption isotherms, and the adsorption kinetics was described by the logistic model [24]. The activation energy values and thermodynamic parameters indicated that the adsorption process was physical, spontaneous, and exothermic.

Native and $\mathrm{NaOH}$-modified shells of muscat pumpkin (Cucurbita moschata Duchesne ex Poir) seeds were studied to remove $\mathrm{Cu}^{2+}$ ions from simulated solutions. It was determined that the maximum sorption capacities were 14.96 and $14.32 \mathrm{mg} / \mathrm{g}$ for native and modified pumpkin seed shells at an initial $\mathrm{Cu}^{2+}$ concentration of $553 \mathrm{mg} / \mathrm{g}, \mathrm{pH}=7$, solution temperature of $29^{\circ} \mathrm{C}$, and dosage of sorption materials of $40 \mathrm{~g} / \mathrm{dm}^{3}$. It was determined that the Langmuir equation more accurately describes adsorption isotherms, and the adsorption kinetics follows the pseudo-second-order model. The calculated adsorption activation energies for the initial and the modified pumpkin seed shells (42.4 and $69.42 \mathrm{~kJ} / \mathrm{mol}$, respectively) indicate chemisorption [25].

Methylene Blue dye adsorption by native shells of common pumpkin seeds (Cucurbita pepo L.) at $30^{\circ} \mathrm{C}$ was studied. It was found that the maximum adsorption capacity for this dye is $141.92 \mathrm{mg} / \mathrm{g}$. The adsorption isotherm is most adequately described by the multilayer adsorption model $(\mathrm{R} 2=0.999)$. The process kinetics is more accurately described by the pseudo-first-order model. The process mechanism study showed that the initial period is characterized by diffusion. In the future, diffusion into meso- and macropores of the sorption material is predominant [26].

Reactive Black 5 adsorption from simulated solutions by native shells of common pumpkin seeds was also studied. It was found that the maximum sorption capacity for the said dye is $9.18 \mathrm{mg} / \mathrm{g}$ when the dosage of pumpkin shells is $1 \mathrm{~g} / \mathrm{dm}^{3}, \mathrm{pH}=2$, and the contact time is 30 minutes at the initial pollutant concentration of $100 \mathrm{mg} / \mathrm{dm}^{3}$. The Langmuir equation describes the adsorption isotherm, and the process kinetics is described by a pseudo-secondorder model [27].

Erythrosine B adsorption process was studied on native shells of pumpkin seeds. It was determined that this dye's maximum sorption capacity is $16.4 \mathrm{mg} / \mathrm{g}$ at its initial concentration of $400 \mathrm{mg} / \mathrm{dm}^{3}$. The adsorption mechanism is defined by the diffusion of erythrosine molecules into the particles of pumpkin seed shells, depending on the pore size [28].

Methyl orange adsorption by ethanolamine- and hydrochloric acid-modified pumpkin seed shells was studied. The maximum sorption capacity was $200.3 \mathrm{mg} / \mathrm{g}$ at $318 \mathrm{~K}, \mathrm{pH}=3$, and $\mathrm{T}=120$ minutes. The Sips model most accurately describes the adsorption isotherms in the temperature range of $298-318 \mathrm{~K}$, and the kinetics of the process follows the pseudo-secondorder model [29]. The calculated thermodynamic parameters $\left(\Delta \mathrm{G}^{\mathrm{o}}=-2.761,-4.473\right.$, and $5.201 \mathrm{~kJ} / \mathrm{mol}$ at temperatures of 298,308 , and $318 \mathrm{~K}$, respectively, $\Delta \mathrm{H}^{\mathrm{o}}=33.37 \mathrm{~kJ} / \mathrm{mol}$, and $\Delta \mathrm{S}^{0}$ $=123 \mathrm{~J} / \mathrm{mol} \cdot \mathrm{K}$ ) indicate spontaneous endothermic physical adsorption.

2,4,6-trichlorophenol was removed from aqueous solutions using four sorption materials from agricultural raw material processing waste, including pumpkin seed shells [30]. The maximum sorption capacity of pumpkin seed shells was determined for the said reagent, $41.5 \mathrm{mg} / \mathrm{g}$. At an initial concentration of 2,4,6-trichlorophenol amounting to $0.025 \mathrm{mmol} / \mathrm{dm}^{3}$, $\mathrm{pH}=6$, and a solution temperature of $25^{\circ} \mathrm{C}$, the degree of pollutant removal ranged from 12 to $68 \%$ with an increase in the mass of pumpkin seed shells from 5 to $25 \mathrm{~g} / \mathrm{dm}^{3}$. It was found that 
the adsorption isotherm is most accurately described by the Freundlich equation $\left(R^{2}=0.9939\right)$, and the kinetics of the process follows the pseudo-second-order model.

Adsorption of suspended coal dust substances from wastewater was studied using pumpkin seed shells modified with phosphoric acid or ammonium chloride [31]. The adsorption isotherm is adequately described $\left(\mathrm{R}^{2}>0.99\right)$ by the Langmuir equation, and the process kinetics is described by the pseudo-second-order equation.

There are several reports on the use of biomass and seed shells of the so-called "fluted gourd" (Telfairia occidentalis), a plant from the Cucurbitaceae family, which is widely distributed in West Africa, as a sorption material. The fluted gourd fruits are very large - from 16 to $105 \mathrm{~cm}$ in length and an average of $9 \mathrm{~cm}$ in diameter, but inedible. There are up to 196 seeds in large gourds, the length of which is usually from 3.4 to $4.9 \mathrm{~cm}$. Edible is the seeds of fluted gourds, which consist of $27 \%$ of raw protein and $53 \%$ of fat [32].

The native and carboxylated rind of fluted gourd of various sizes was studied to extract $\mathrm{Cd}^{2+}, \mathrm{Hg}^{2+}, \mathrm{Pb}^{2+}$, and $\mathrm{Zn}^{2+}$ ions [21]. At the initial concentration of these ions of $100 \mathrm{mg} / \mathrm{dm}^{3}$, the dosage of the sorption material of $1.2 \mathrm{~g} / \mathrm{dm}^{3}, \mathrm{pH}=7.5$ and 1-hour contact, the degree of metal ions removal by native Telfairia occidentalis rind was 90\%, 62\%, 95\%, and 82\%, respectively. For a carboxylated sample of fluted gourd rind, these ions' removal rates were $63 \%, 42 \%, 78 \%$, and $79 \%$, respectively.

Fluted gourd (Telfairia occidentalis) biomass modified with sulfonyl acetic acid was studied as a sorption material for removing $\mathrm{Cd}^{2+}, \mathrm{Pb}^{2+} \mathrm{Zn}^{2+}$ ions from simulated solutions. It was found that the native biomass of fluted gourd powder has an area of $33 \mathrm{~m}^{2} / \mathrm{g}$; after modification, the sample area increased to $90 \mathrm{~m}^{2} / \mathrm{g}$ [33]. It was found that the best sorption parameters are achieved at $\mathrm{pH}=4$, solution temperature $40{ }^{\circ} \mathrm{C}$, and the contact time more than 30 minutes. The maximum sorption capacities for $\mathrm{Cd}^{2+}, \mathrm{Pb}^{2+}$, and $\mathrm{Zn}^{2+}$ ions were determined using the Langmuir equation for native fluted gourd biomass: 9.79, 9.54, and $13.33 \mathrm{mg} / \mathrm{g}$, respectively, and for modified Telfairia occidentalis biomass: $11.34,9.26$, and $17.18 \mathrm{mg} / \mathrm{g}$, respectively. The calculated thermodynamic parameters indicate exothermic physical adsorption.

Adsorption of divalent metal ions ( $\mathrm{Hg}, \mathrm{Rh}, \mathrm{Pt}$, and $\mathrm{Pd}$ ) by fluted gourd biomass was studied. It was determined that the maximum sorption capacity for these metals was $9.89 \mathrm{mg} / \mathrm{g}$, $9.81 \mathrm{mg} / \mathrm{g}, 10.59 \mathrm{mg} / \mathrm{g}$, and $6.84 \mathrm{mg} / \mathrm{g}$, respectively, and correlates with the size of the ions of these metals [34]. This statement is confirmed by experiments on adsorption of $\mathrm{Al}^{3+}, \mathrm{Co}^{2+}$, and $\mathrm{Ag}^{+}$ions by the biomass of fluted gourd. The maximum sorption capacity for these ions was $16.98 \mathrm{mg} / \mathrm{g}, 10.34 \mathrm{mg} / \mathrm{g}$, and $8.03 \mathrm{mg} / \mathrm{g}$, respectively, and correlated with the size of metal ions. A decrease in the size of the metal ion $\left(\mathrm{Al}^{3+}=0.52 \mathrm{~A} ; \mathrm{Co}^{2+}=0.78 \mathrm{~A}^{2}\right.$ and $\left.\mathrm{Ag}^{+}=1.26 \mathrm{~A}\right)$ resulted in an increase of sorption parameters [34]. It was determined that the metal ion adsorption isotherms are well described $\left(\mathrm{R}^{2}>0.99\right)$ by the Langmuir equation $[33,34]$ and the Freundlich equation [35].

Besides, native and 2-mercaptoethanoic acid-modified Telfairia occidentalis biomass was studied as a sorption material for removing $\mathrm{Ni}^{2+}$ ions from simulated solutions. It was found that the native biomass maximum sorption capacity for $\mathrm{Ni}^{2+}$ ions was $12.69 \mathrm{mg} / \mathrm{g}$. That of biomass treated with 0.5 and $1.0 \mathrm{~N}$ solutions of 2-mercaptoethanoic acids was 40.0 and $42.19 \mathrm{mg} / \mathrm{g}$, respectively [36]. It was found that at the initial $\mathrm{Ni}^{2+}$ ions concentration of $50 \mathrm{mg} / \mathrm{dm}^{3}$ in the solution, the highest removal rate of the latter was achieved at $\mathrm{pH}=4-5.5$, a temperature of $30^{\circ} \mathrm{C}$ and 10 minutes of contact. The calculated thermodynamic parameters show that the process is physical and exothermic. 
The removal of Congo red, Bromocresol green, and Indigo carmine dyes was studied using dried biomass of fruits of edible chayote (Sechium edule, an edible plant from the Sechium genus, the gourd family (Cucurbitaceae)) and fig-leaved gourd (Cucurbita ficifolia) in a dosage of 10 to $30 \mathrm{~g} / \mathrm{dm}^{3}$. It was determined that chayote fruits' biomass contributes to the complete discoloration in solutions after 24 hours of contact. The process kinetics follows the pseudo-second-order model [37].

\section{The Use of Watermelon Fruit Waste as Sorption Materials for Removal of Metal Ions and Dyes from Aqueous Media}

A bit more publications are devoted to studying the use of watermelon fruit waste as effective sorption materials. Watermelon (Citrullus lanatus) is an annual herbaceous plant, a watermelon (Citrullus) species of the gourd family (Cucurbitaceae). The fruit is a pumpkin, spherical, oval, flattened, or cylindrical; the rind color is from white and yellow to dark green with stripes and spots; the flesh is pink, red, crimson, rarely white and yellow [38]. The watermelon fruit pulp contains 5.5 to $13 \%$ of easily digestible sugars (glucose, fructose, and sucrose). By the time of maturation, glucose and fructose predominate, sucrose accumulates during the storage of watermelon. The pulp contains pectins $-0.68 \%$, proteins $-0.7 \%$; calcium $-14 \mathrm{mg} / \%$ (mg per $100 \mathrm{~g}$ ), magnesium $-224 \mathrm{mg} / \%$, sodium $-16 \mathrm{mg} / \%$, potassium $64 \mathrm{mg} / \%$, phosphorus - $7 \mathrm{mg} / \%$; vitamins - thiamine, riboflavin, folic acid, carotene -0.1 $0.7 \mathrm{mg} / \%$, ascorbic acid $-0.7-20 \mathrm{mg} / \%$ [38].

People use watermelon pulp mainly for food. Its main waste is the rind. The latter does not contain amino acids and includes some vitamins: retinol (vitamin A) - 51.5mg/100g, ascorbic acid (vitamin C) $-7.23 \mathrm{mg} / 100 \mathrm{~g}$, as well as vitamins $\mathrm{B}_{1}, \mathrm{~B}_{2}, \mathrm{~B}_{3}$ and $\mathrm{B}_{6}$ in the amount of $0.03,0.02,0.04$ and $0.04 \mathrm{mg} / 100 \mathrm{~g}$, respectively [38] . Watermelon rind also contains elements ranging from $4 \mathrm{mg} / \mathrm{kg}$ of copper to $1,297 \mathrm{mg} / \mathrm{kg}$ of phosphorus [39]. Watermelon rind is widely used in various industries [19].

One of the watermelon rind applications uses it as a sorption material to remove various pollutants from water environments [40-68].

Heavy metal ions. The presence of biopolymers with different functional groups in watermelon rind, e.g., $\mathrm{C}(\mathrm{O}) \mathrm{OH}$ in pectin, $-\mathrm{OH}$ in cellulose, $-\mathrm{NH}_{2}$ in proteins, etc., should contribute to metal ions removal. The sorption characteristics and the conditions for conducting experiments to study the removal of metal ions using native and modified watermelon rind are shown below (Table 1).

Table 1. Experiment conditions and metal ions adsorption characteristics of the native and modified watermelon rind.

\begin{tabular}{|c|c|c|c|c|}
\hline Metal ion & Experiment conditions & Adsorption characteristics & Note & Source \\
\hline $\mathrm{As}^{3+}$ & $\begin{array}{l}\mathrm{D}_{\text {sorp }}<0.2 \mathrm{~mm}, \mathrm{C}_{0}=4 \mathrm{mg} / \mathrm{dm}^{3}, \\
\mathrm{pH}=7.2-8.2, \mathrm{t}=60 \mathrm{~min}, \mathrm{DS}= \\
1 \mathrm{~g} / \mathrm{dm}^{3}, \text { modified with xanthate } \\
\text { and citric acid }\end{array}$ & $\begin{array}{l}\mathrm{A}=\sim 4 \mathrm{mg} / \mathrm{g}, \text { removal rate }- \\
99 \%\end{array}$ & $\begin{array}{l}\text { the Langmuir model, } \\
\text { pseudo-second } \\
\text { kinetics }\end{array}$ & {$[40]$} \\
\hline $\mathrm{As}^{5+}$ & $\begin{array}{l}\mathrm{D}_{\text {sorp }}<0.2 \mathrm{~mm}, \mathrm{C}_{0}=4 \mathrm{mg} / \mathrm{dm}^{3}, \\
\mathrm{pH}=4.5-5.3, \mathrm{t}=60 \mathrm{~min}, \mathrm{DS}= \\
1 \mathrm{~g} / \mathrm{dm}^{3}, \text { modified with xanthate } \\
\text { and citric acid }\end{array}$ & $\begin{array}{l}\mathrm{A}=\sim 4 \mathrm{mg} / \mathrm{g}, \text { removal rate }- \\
98 \%\end{array}$ & $\begin{array}{l}\text { the Langmuir model, } \\
\text { pseudo-second } \\
\text { kinetics }\end{array}$ & {$[40]$} \\
\hline $\mathrm{Cd}^{2+}$ & $\begin{array}{l}\mathrm{C}_{0}=50-200 \mathrm{ppm}, \mathrm{pH}=2-8 \\
50 \mathrm{rpm}, \mathrm{t}=5-120 \mathrm{~min}, \mathrm{DS}= \\
0.5-5.0 \mathrm{~g} / \mathrm{dm}^{3}\end{array}$ & $\mathrm{~A}_{\max }=63.29 \mathrm{mg} / \mathrm{g}$ & $\begin{array}{l}\text { the Langmuir model }\left(\mathrm{R}^{2}=\right. \\
0.995), \quad \text { pseudo-second } \\
\text { order kinetics }\left(\mathrm{R}^{2}=1.0\right)\end{array}$ & {$[41]$} \\
\hline $\mathrm{Cr}^{3+}$ & $\begin{array}{l}\mathrm{C}_{0}=50-300 \mathrm{ppm}, \mathrm{pH}=2-8 \\
150 \mathrm{rpm}, \mathrm{t}=30 \mathrm{~min}, \mathrm{DS}=0.5- \\
5.0 \mathrm{~g} / \mathrm{dm}^{3}\end{array}$ & $\begin{array}{l}\mathrm{A}_{\max }=172.6 \mathrm{mg} / \mathrm{g}, \text { maximum } \\
\text { removal rate }-90.8 \%(\mathrm{pH}=3 \\
\left.\mathrm{C}_{0}=50 \mathrm{ppm}, \mathrm{DS}=1.5 \mathrm{~g} / \mathrm{dm}^{3}\right)\end{array}$ & $\begin{array}{l}\text { the Langmuir model }\left(\mathrm{R}^{2}=\right. \\
0.9989), \quad \text { pseudo-second } \\
\text { order kinetics; } \Delta \mathrm{G}^{\mathrm{o}}=\text { - }\end{array}$ & \\
\hline
\end{tabular}




\begin{tabular}{|c|c|c|c|c|}
\hline Metal ion & Experiment conditions & Adsorption characteristics & Note & Source \\
\hline & & & $\begin{array}{l}3.330, \quad-2.234, \quad \text { and } \quad- \\
2.165 \mathrm{~kJ} / \mathrm{mol}(303,313 \text {, and } \\
323 \quad \mathrm{~K}), \Delta \mathrm{H}^{\mathrm{o}}=\quad- \\
3.741 \mathrm{~kJ} / \mathrm{mol}, \quad \Delta \mathrm{S}^{\mathrm{o}}=\quad- \\
582 \mathrm{~J} / \mathrm{mol} \cdot \mathrm{K}\end{array}$ & {$[42]$} \\
\hline $\mathrm{Cu}^{2+}$ & $\begin{array}{l}\mathrm{C}_{0}=10 \mathrm{mg} / \mathrm{dm}^{3} ; \mathrm{pH}=6.48 ; \mathrm{DS} \\
=1 \mathrm{~g} / \mathrm{dm}^{3} ; \mathrm{t}=10 \mathrm{~h} ; 125 \mathrm{rpm} ; \mathrm{T}= \\
20{ }^{\circ} \mathrm{C}\end{array}$ & $\mathrm{A}_{\max }=5.73 \mathrm{mg} / \mathrm{g}$ & & {$[43]$} \\
\hline $\mathrm{Cu}^{2+}$ & $\begin{array}{l}\mathrm{C}_{0}=5-20 \mathrm{mg} / \mathrm{dm}^{3}, \mathrm{DS}=0,5- \\
2 \mathrm{~g} / \mathrm{dm}^{3}, \mathrm{pH}=2-10, \mathrm{t}=1-4 \mathrm{~h}\end{array}$ & $A_{\max }=9.54 \mathrm{mg} / \mathrm{g}$ & the Freundlich model & {$[44]$} \\
\hline $\mathrm{Cu}^{2+}$ & $\begin{array}{l}\mathrm{C}_{0}=10 \mathrm{mg} / \mathrm{dm}^{3}, \text { DS }=0.02- \\
2 \mathrm{~g} / \mathrm{dm}^{3}, \mathrm{~T}=26^{\circ} \mathrm{C}, 150 \mathrm{rpm}, \mathrm{t}= \\
1-200 \mathrm{~min},\end{array}$ & $\mathrm{~A}_{\max }=111.1 \mathrm{mg} / \mathrm{g}$ & $\begin{array}{l}\text { the Langmuir } \begin{array}{r}\text { model, } \\
\text { order }\end{array} \\
\text { pseudo-second } \\
\text { kinetics; } \Delta \mathrm{G}^{\mathrm{o}}=-4.584,- \\
4.916, \text { and }-5.249 \mathrm{~kJ} / \mathrm{mol} \\
(303,313 \text { and } 323 \mathrm{~K}), \Delta \mathrm{H}^{\mathrm{o}} \\
=-5.494 \mathrm{~kJ} / \mathrm{mol}, \Delta \mathrm{S}^{\mathrm{o}}= \\
33.26 \mathrm{~J} / \mathrm{mol} \cdot \mathrm{K}\end{array}$ & {$[45]$} \\
\hline $\mathrm{Cu}^{2+}$ & $\begin{array}{l}\mathrm{C}_{0}=10-40 \mathrm{mg} / \mathrm{dm}^{3}, \mathrm{DS}=0,2- \\
2 \mathrm{~g} / \mathrm{dm}^{3}, \mathrm{~T}=30{ }^{\circ} \mathrm{C}, \mathrm{t}=1-60 \\
\text { min, } \mathrm{pH}=2-8, \quad 60-130 \mathrm{~W} \\
\text { ultrasound treatment. }\end{array}$ & $\begin{array}{l}\mathrm{A}_{\max }=31.25 \mathrm{mg} / \mathrm{g}\left(\mathrm{CA}(\mathrm{OH})_{2}\right. \\
\text { modification), } \quad \mathrm{A}_{\max }= \\
27.03 \mathrm{mg} / \mathrm{g} \quad \text { (citric acid } \\
\text { modification). }\end{array}$ & $\begin{array}{l}\text { the Langmuir model, } \\
\text { pseudo-second-order } \\
\text { kinetics. }\end{array}$ & {$[46]$} \\
\hline $\mathrm{Ni}^{2+}$ & $\begin{array}{l}\mathrm{C}_{0}=10-60 \mathrm{Mr} / \mathrm{dm}^{3}, \mathrm{pH}=2-10 \\
\mathrm{DS}=1-5 \mathrm{~g} / \mathrm{dm}^{3}, \mathrm{t}=2-120 \mathrm{~min} \\
\mathrm{~T}=30^{\circ} \mathrm{C}\end{array}$ & $\mathrm{S}=150.6 \mathrm{~m} 2 / \mathrm{g}, \mathrm{A}_{\text {мах }}=38.98 \mathrm{M \Gamma} / \Gamma$ & $\begin{array}{l}\text { the Langmuir model, } \\
\text { pseudo-second order } \\
\text { kinetics, Dumwald- } \\
\text { Wagner diffusion model } \\
\left(\mathrm{R}^{2}=0.9795\right) \text {. }\end{array}$ & {$[47]$} \\
\hline $\mathrm{NH}_{4}^{+}$ & $\begin{array}{l}\mathrm{C}_{0}=1-50 \mathrm{M} \Gamma / \mathrm{dm}^{3}, \mathrm{pH}=5-7, \mathrm{DS} \\
=10-40 \mathrm{~g} / \mathrm{dm}^{3}, \mathrm{t}=1-40 \mathrm{~min}, \\
\mathrm{KOH}, \quad \mathrm{NaOH}, \\
\begin{array}{l}\left(20 \mathrm{mmol} / \mathrm{dm}_{2}^{3}\right) \\
\text { modification }\end{array}\end{array}$ & $\begin{array}{l}\mathrm{A}_{\text {мах }}=1.24 \mathrm{mg} / \mathrm{g} \quad \text { (native } \\
\text { watermelon rind), } 1.21 \mathrm{mg} / \mathrm{g} \\
(\mathrm{KOH}), 1.21 \quad(\mathrm{NaOH}), 1.23 \\
\left(\mathrm{H}_{2} \mathrm{SO}_{4}\right) .\end{array}$ & & {$[48]$} \\
\hline $\mathrm{NH}_{4}^{+}$ & $\begin{array}{lr}\mathrm{C}_{0}=1-50 \mathrm{mg} / \mathrm{dm}^{3}, & \mathrm{pH}=7, \\
\mathrm{KOH}, \quad \mathrm{NaOH}, & \mathrm{H}_{2} \mathrm{SO}_{4} \\
\left(20 \mathrm{mmol} / \mathrm{dm}^{3}\right) & \text { solutions } \\
\text { modification } & \\
\end{array}$ & $\begin{array}{l}\mathrm{A}_{\text {мах }}=1.22 \mathrm{mg} / \mathrm{g} \quad \text { (native } \\
\text { watermelon rind), } 1.21 \mathrm{mg} / \mathrm{g} \\
(\mathrm{KOH}), 1.19(\mathrm{NaOH}), 1.19 \\
\left(\mathrm{H}_{2} \mathrm{SO}_{4}\right) .\end{array}$ & $\begin{array}{l}\text { the Langmuir model }\left(\mathrm{R}^{2}=\right. \\
1.0 \text { in all cases }), \text { pseudo- } \\
\text { second order kinetics }\left(\mathrm{R}^{2}=\right. \\
1.0 \text { in all cases })\end{array}$ & [49] \\
\hline $\mathrm{Zn}^{2+}$ & $\begin{array}{l}\mathrm{C}_{0}=100 \mathrm{ppm} / \mathrm{dm}^{3}, \mathrm{pH}=6, \mathrm{~T}= \\
60{ }^{0} \mathrm{C}, 150 \mathrm{rpm}, \mathrm{DS}=4 \mathrm{~g} / \mathrm{dm}^{3} \text {, } \\
\text { modification with citric acid } \\
\text { solutions, } \mathrm{H}_{2} \mathrm{SO}_{4}, \mathrm{Ca}(\mathrm{OH})_{2} \text {, } \\
\mathrm{NaOH}\end{array}$ & 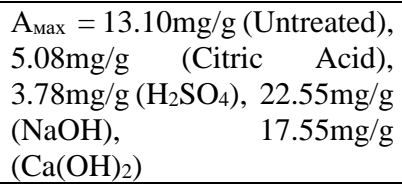 & & {$[50]$} \\
\hline $\mathrm{Zn}^{2+}$ & $\begin{array}{l}\mathrm{C}_{0}=400 \mathrm{mg} / \mathrm{dm}^{3}, \mathrm{pH}=8, \mathrm{DS}= \\
1.5 \mathrm{mg} / \mathrm{dm}^{3}, \mathrm{t}=30 \mathrm{~min}\end{array}$ & & & {$[51]$} \\
\hline
\end{tabular}

$\mathrm{C}_{0}$ is the initial concentration of pollutants in solutions, DS is the dosage of the sorption material, $\mathrm{T}$ is the experiment temperature, $\mathrm{t}$ is the adsorption time, and $\mathrm{A}_{\max }$ is the maximum adsorption capacity.

As follows from the data shown in Table 1, experiments on metal ions and ammonium nitrogen adsorption were carried out under various conditions. This circumstance contributed to the fact that the resulting adsorption parameters have a wide range of values. In all studies, the adsorption isotherms of native and modified watermelon rind are most accurately described by the Langmuir equation. The process kinetics follows the pseudo-second-order model.

Experiments were done in which adsorption of two or three metal ions by watermelon rind was studied under the same conditions. For example, $\mathrm{Co}^{2+}$ and $\mathrm{Ni}^{2+}$ ions adsorption by dried watermelon rind was studied under equal conditions - with the initial metal ions concentration of $50 \mathrm{mg} / \mathrm{dm}^{3}, \mathrm{pH}=5$, contact time 3 minutes, and the sorption material dosage of 2.0 and $2.5 \mathrm{~g} / \mathrm{dm}^{3}$, respectively. It was found that the maximum sorption capacity for $\mathrm{Co}^{2+}$ ions was $23.3 \mathrm{mg} / \mathrm{g}$, and for $\mathrm{Ni}^{2+}$ ions $-35.3 \mathrm{mg} / \mathrm{g}$ [52].

The maximum sorption capacity of dried watermelon rind treated with $0.1 \mathrm{~mol} \mathrm{HCl}$ solution for $\mathrm{Cu}^{2+}$ and $\mathrm{Pb}^{2+}$ ions was $39.2 \mathrm{mg} / \mathrm{g}$ and $116.2 \mathrm{mg} / \mathrm{g}$, respectively [53]. In another study, it was found that under comparable experimental conditions, the maximum sorption 
capacity for $\mathrm{Pb}^{2+}$ ions was $1.4 \mathrm{mg} / \mathrm{g}$. For $\mathrm{Zn}^{2+}$ ions, it was $23.3 \mathrm{mg} / \mathrm{g}$ [54] with a degree of purification of $77.3 \%$ and $90.3 \%$, respectively [55].

A study was also made of the process of adsorption of $\mathrm{Cu}^{2+}, \mathrm{Zn}^{2+}$, and $\mathrm{Pb}^{2+}$ ions by native dried watermelon rind. At the initial concentration of all metal ions in a $10 \mathrm{mg} / \mathrm{dm}^{3}$ solution, the efficiency of removal of these ions is as follows: $\mathrm{Pb}>\mathrm{Cu}>\mathrm{Zn}$. The maximum sorption capacity calculated from the adsorption isotherms using the Langmuir equation was $98.06,6.28$, and $6.85 \mathrm{mg} / \mathrm{g}$, respectively [56]. Watermelon rind treated with $5 \% \mathrm{HNO}_{3}$ solution was used to extract heavy metal ions from aqueous solutions. At an initial $\mathrm{Zn}^{2+}$ $2.5 \mathrm{mg} / \mathrm{dm}^{3}, \mathrm{Fe}^{3+} 0.9 \mathrm{mg} / \mathrm{dm}^{3}$, and $\mathrm{Pb}^{2+} 0.09 \mathrm{mg} / \mathrm{dm}^{3}$ concentration, the final concentrations were $0.36 \mathrm{mg} / \mathrm{dm}^{3}$ (recovery rate $\left.85.6 \%\right), 0.35 \mathrm{mg} / \mathrm{dm}^{3}(61.1 \%)$, and $0.04 \mathrm{mg} / \mathrm{dm}^{3}(55.6 \%)$, respectively [57].

Watermelon rinds have also been studied as a biosorbent for removing dyes from aqueous media. Information about the experimental conditions and the results on the adsorption of various dyes by watermelon rind are shown in Table 2 .

Table 2. Experiment conditions and dyes adsorption characteristics of the native and modified watermelon rind.

\begin{tabular}{|c|c|c|c|c|}
\hline Dye & Experiment conditions & Adsorption characteristics & Note & Source \\
\hline $\begin{array}{l}\text { Methylene } \\
\text { blue }\end{array}$ & $\begin{array}{l}\mathrm{C}_{0}=50-400 \mathrm{mg} / \mathrm{dm}^{3}, \mathrm{pH}=3- \\
10, \mathrm{t}=0-180 \mathrm{~min}, \mathrm{DS}=0.2- \\
2.0 \mathrm{~g} / \mathrm{dm}^{3}, 125 \mathrm{rpm}\end{array}$ & $\mathrm{A}_{\max }=188.68 \mathrm{mg} / \mathrm{g}$ & $\begin{array}{l}\text { the Langmuir and } \\
\text { Tyomkin models, pseudo- } \\
\text { second order kinetics; } \\
\Delta \mathrm{G}^{\mathrm{o}}=-5.811,-5.796 \text {, and } \\
-5.781 \mathrm{~kJ} / \mathrm{mol}(303,313 \\
\text { and } 323 \mathrm{~K}), \Delta \mathrm{H}^{\mathrm{o}}=- \\
6.267 \mathrm{~kJ} / \mathrm{mol}, \Delta \mathrm{S}^{\mathrm{o}}=- \\
1503 \mathrm{~J} / \mathrm{mol} \cdot \mathrm{K}\end{array}$ & {$[58,59]$} \\
\hline Brilliant green & $\begin{array}{l}\text { Orthophosphoric } \quad \text { acid } \\
\text { modification }\end{array}$ & $\begin{array}{l}\mathrm{A}_{\text {мах }}=92.6 \mathrm{mg} / \mathrm{g} \text { (native } \\
\text { rind), } \mathrm{A}_{\text {мах }}=188.6 \mathrm{mg} / \mathrm{g} \\
\text { (modified) }\end{array}$ & $\begin{array}{l}\text { the Langmuir } \begin{array}{r}\text { model, } \\
\text { pseudo-second } \\
\text { kinetics }\end{array} \\
\text { order }\end{array}$ & {$[60]$} \\
\hline Congo Red & $\begin{array}{l}\mathrm{C}_{0}=5-300 \mathrm{mg} / \mathrm{dm}^{3}, \mathrm{pH}=2-12 \\
\mathrm{t}=5-240 \mathrm{~min}, \quad \mathrm{DS}=2- \\
10 \mathrm{~g} / \mathrm{dm}^{3}, \mathrm{~T}=25^{\circ} \mathrm{C}, 300 \mathrm{rpm}\end{array}$ & $\mathrm{A}_{\max }=24.75 \mathrm{mg} / \mathrm{g}$ & $\begin{array}{l}\text { the Langmuir model }\left(\mathrm{R}^{2}=\right. \\
0.9998)\end{array}$ & {$[61]$} \\
\hline Methyl orange & $\begin{array}{l}\mathrm{C}_{0}=300 \mathrm{mg} / \mathrm{dm}^{3}, \mathrm{pH}=2-12, \mathrm{t} \\
=5-240 \mathrm{~min}, \mathrm{DS}=2 \mathrm{~g} / \mathrm{dm}^{3}, \mathrm{~T} \\
=303-333 \mathrm{~K}, 150 \mathrm{rpm}\end{array}$ & $\mathrm{A}_{\max }=24.86 \mathrm{mg} / \mathrm{g}$ & $\begin{array}{l}\text { pseudo-second } \\
\text { kinetics }\end{array}$ & {$[62]$} \\
\hline $\begin{array}{l}\text { Remazol } \\
\text { Brilliant Blue }\end{array}$ & $\begin{array}{l}\mathrm{C}_{0}=25-150 \mathrm{mg} / \mathrm{dm}^{3}, \mathrm{pH}=2- \\
10, \mathrm{t}=24 \mathrm{~h}, \mathrm{DS}=0.5- \\
1.5 \mathrm{~g} / \mathrm{dm}^{3}, \mathrm{~T}=25^{\circ} \mathrm{C}, 100 \mathrm{rpm}\end{array}$ & $\mathrm{A}_{\max }=29.4 \mathrm{mg} / \mathrm{g}$ & $\begin{array}{l}\text { The Freundlich equations } \\
\left(\mathrm{R}^{2}=0.945\right) \text {, pseudo- } \\
\text { second order kinetics }\left(\mathrm{R}^{2}\right. \\
=0.99)\end{array}$ & {$[63]$} \\
\hline Alizarin Red-S & $\begin{array}{l}\mathrm{C}_{0}=5-40 \mathrm{ppm}, \mathrm{pH}=1-10, \mathrm{t}= \\
5-80 \mathrm{~min}, \mathrm{DS}=0.5-1.5 \mathrm{~g} / \mathrm{dm}^{3}, \\
\mathrm{~T}=20-70^{\circ} \mathrm{C}, 100 \mathrm{rpm}\end{array}$ & $\begin{array}{l}\text { The maximum dye removal } \\
\text { efficiency is } 94.0 \% . A_{\max }= \\
79.6 \mathrm{mg} / \mathrm{g}\end{array}$ & $\begin{array}{l}\text { the Tyomkin model }\left(\mathrm{R}^{2}=\right. \\
0.987), \text { pseudo-second- } \\
\text { order kinetics }\left(\mathrm{R}^{2}=\right. \\
0.903) . \Delta \mathrm{G}^{\mathrm{o}}= \\
15.40 \mathrm{~kJ} / \mathrm{mol}\end{array}$ & {$[64]$} \\
\hline Emerald green & & $\mathrm{A}_{\max }=2.1 \mathrm{mg} / \mathrm{g}$ & $\Delta \mathrm{G}^{\mathrm{o}}=-2.785 \mathrm{~kJ} / \mathrm{mol}$ & [65] \\
\hline $\begin{array}{l}\text { Methyl } \\
\text { Orange, } \\
\text { Rhodamine B }\end{array}$ & $\begin{array}{l}\mathrm{C}_{0}=10-310 \mathrm{mg} / \mathrm{dm}^{3}, \mathrm{pH}=2- \\
12, \mathrm{t}=5-120 \mathrm{~min} \mathrm{~h}, \mathrm{DS}=1- \\
20 \mathrm{~g} / \mathrm{dm}^{3}, \mathrm{~T}=25^{\circ} \mathrm{C}, 300 \mathrm{rpm} . \\
\text { Treatment with sulfuric acid } \\
\text { and annealing at } 300^{\circ} \mathrm{C} \text { for } 3 \\
\text { hours. }\end{array}$ & Dye removal rate $\sim 100 \%$ & $\begin{array}{l}\text { pseudo-second order } \\
\text { kinetics }\end{array}$ & {$[66]$} \\
\hline $\begin{array}{l}\text { Basic red 2, } \\
\text { Orange } G\end{array}$ & $\begin{array}{l}\mathrm{C}_{0}=140 \mathrm{mg} / \mathrm{dm}^{3}, \mathrm{pH}=2-11, \mathrm{t} \\
=180 \mathrm{~min} \mathrm{~h}, \mathrm{DS}=0.35- \\
25 \mathrm{~g} / \mathrm{dm}^{3}, \mathrm{~T}=293-318 \mathrm{~K}, \\
150 \mathrm{rpm} .\end{array}$ & $\begin{array}{l}\mathrm{A}_{\text {мах }}=73,53 \mathrm{mg} / \mathrm{g} \text { (Basic red } \\
2), \quad \mathrm{A}_{\text {мах }}=68.14 \mathrm{mg} / \mathrm{g} \\
(\text { Orange } \mathrm{G})\end{array}$ & $\begin{array}{l}\text { Both isotherms are } \\
\text { described by the } \\
\text { Langmuir model; the } \\
\text { kinetics is most accurately } \\
\text { described by the model of } \\
\text { Fractal-like. } \\
\Delta \mathrm{G}^{\mathrm{o}}=-23.62-- \\
21.23 \mathrm{~kJ} / \mathrm{mol}(293-318 \mathrm{~K}) \text {, } \\
\Delta \mathrm{H}^{\mathrm{o}}=0.09 \mathrm{~kJ} / \mathrm{mol}, \Delta \mathrm{S}^{\mathrm{o}}=- \\
50.04 \mathrm{~J} / \mathrm{mol} \cdot \mathrm{K}(\mathrm{Basic} \mathrm{red} \\
2) ; \mathrm{G}^{\mathrm{o}}=-22.39-- \\
24.73 \mathrm{~kJ} / \mathrm{mol}(293-318 \mathrm{~K}) \text {, }\end{array}$ & {$[67]$} \\
\hline
\end{tabular}




\begin{tabular}{|c|c|c|c|c|}
\hline Dye & Experiment conditions & Adsorption characteristics & Note & Source \\
\hline & & & $\begin{array}{l}\Delta \mathrm{H}^{\mathrm{o}}=-0.94 \mathrm{~kJ} / \mathrm{mol}, \Delta \mathrm{S}^{\mathrm{o}}= \\
5.127 \mathrm{~J} / \mathrm{mol} \cdot \mathrm{K}(\text { Orange } \mathrm{G})\end{array}$ & \\
\hline $\begin{array}{l}\text { Fluorescein, } \\
\text { Eosin }\end{array}$ & $\begin{array}{l}\mathrm{C}_{0}=50 \mathrm{mg} / \mathrm{dm}^{3}, \mathrm{pH}=1-7, \mathrm{t}= \\
30 \mathrm{~min} \mathrm{~h}, \mathrm{DS}=4-40 \mathrm{~g} / \mathrm{dm}^{3}, \mathrm{~T}= \\
30{ }^{\circ} \mathrm{C}, \quad 100 \mathrm{rpm} . \quad \text { Treatment } \\
\text { with } 0.1 \mathrm{~N} \mathrm{NaOH} \text { and } 0.1 \mathrm{~N} \\
\mathrm{HNO}_{3}\end{array}$ & $\begin{array}{l}\text { The maximum Fuorescein } \\
\text { removal rate is } 64.83 \% \\
\text { (native), } 81.96 \%\left(\mathrm{HNO}_{3}\right) \text {, } \\
86.33 \% \text { (NaOH); Eosin } \\
\text { removal rate is }-64.74 \% \\
\text { (native), } 76.01 \%-\left(\mathrm{HNO}_{3}\right) \text {, } \\
79.41 \%(\mathrm{NaOH})\end{array}$ & $\begin{array}{l}\text { The adsorption isotherms } \\
\text { are described by the } \\
\text { Langmuir (Fuorescein) } \\
\text { and Freundlich (Eosin) } \\
\text { models. Pseudo-second } \\
\text { order kinetics. } \Delta \mathrm{H}^{\mathrm{o}}=- \\
0.048 \mathrm{~kJ} / \mathrm{mol}, \Delta \mathrm{S}^{\mathrm{o}}= \\
59 \mathrm{j} / \mathrm{mol} \cdot \mathrm{K} \quad(\text { Fuorescein); } \\
\Delta \mathrm{H}^{\mathrm{o}}=-0.051 \mathrm{~kJ} / \mathrm{mol}, \Delta \mathrm{S}^{\mathrm{o}} \\
=34 \mathrm{j} / \mathrm{mol} \cdot \mathrm{K}(\text { Eosin) }\end{array}$ & {$[68]$} \\
\hline $\begin{array}{l}\text { Methylene } \\
\text { Blue, Crystal } \\
\text { violet, } \\
\text { Rhodamine B }\end{array}$ & & 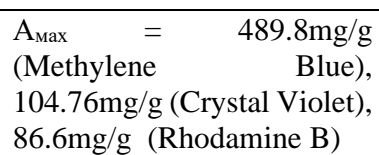 & & [69] \\
\hline
\end{tabular}

Wastewater containing dyes was treated with a composite material made from dried watermelon rind and chitosan. The wastewater had a $\mathrm{pH}$ value of $8.54, \mathrm{COD}-455 \mathrm{mg} / \mathrm{dm} / \mathrm{dm}^{3}$. It was found that the optimal dosage of the composite sorption material was $8 \mathrm{~g} / \mathrm{dm}^{3}$; with $\mathrm{pH}$ $=2$, the efficiency of dye removal was $\sim 100 \%$ [70].

Watermelon processing by-products also include seeds. The latter has also been studied as sorption materials for removing heavy metal ions and dyes from aqueous media. There are reports of $\mathrm{Cd}^{2+}$ ion removal by watermelon seed husks. It was found that at an initial concentration of metal ion $10 \mathrm{mg} / \mathrm{dm}^{3}$, the sorption material completely removes the pollutant; if the initial concentration is increased to $60 \mathrm{mg} / \mathrm{dm}^{3}$, the degree of $\mathrm{Cd}^{2+}$ ion removal is $40.9 \%$. The highest degree of removal at this concentration (47.7\%) is achieved at $\mathrm{pH}=3$ and the amount of watermelon seed husk of $2.5 \mathrm{~g} / \mathrm{dm}^{3}$ [71].

The adsorption of $\mathrm{Cu}^{2+}$ and $\mathrm{Pb}^{2+}$ ions from aqueous solutions using watermelon seed husks was studied. The study involved the determination of the influence of $\mathrm{pH}(2-6)$, biosorbent dose $(0.1-1.0 \mathrm{~g})$, initial metal ion concentration $\left(10-500 \mathrm{mg} / \mathrm{dm}^{3}\right)$, contact time (5$270 \mathrm{~min}$ ), and temperature (293-333K) on the adsorption characteristics. It was found that the Langmuir model most accurately describes the adsorption isotherm, and the kinetics of the process corresponds to the pseudo-second-order model [72]. The adsorption of these two metal ions on the husks of watermelon seeds was found to be a spontaneous and endothermic process.

Watermelon seed husks were studied to remove the Methylene Blue dye from simulated solutions. It was found that the highest removal rate $(87.28 \%)$ of the dye at its initial concentration of $100 \mathrm{mg} / \mathrm{dm}^{3}$ is achieved within 120 minutes of contact at a dosage of the sorption material of $1.5 \mathrm{~g} / \mathrm{dm}^{3}$ and $\mathrm{pH}=10$. It was found that the adsorption isotherm is most adequately described by the Freundlich model [73].

Watermelon seeds themselves have also been studied as a sorption material for $\mathrm{Pb}^{2+}$ ions removal from various water samples (fresh, artesian, sea). $\mathrm{Pb}^{2+}$ ions concentration was $5 \mathrm{mg} / \mathrm{dm}^{3}$. It was shown that the removal rate of these ions was $93.6-96.8 \%$, depending on the type of water. It was found that the maximum sorption capacity of watermelon seeds for $\mathrm{Pb}^{2+}$ ions is $9.64 \mathrm{mg} / \mathrm{g}$ at $\mathrm{pH}=6$. Thermodynamic parameters $\left(\Delta \mathrm{G}^{\mathrm{o}}=-20.96 \mathrm{~kJ} / \mathrm{mol}(333 \mathrm{~K}), \Delta \mathrm{H}^{\mathrm{o}}=\right.$ $18.51 \mathrm{j} / \mathrm{mol}$, and $\left.\Delta \mathrm{S}^{\mathrm{o}}=63.0 \mathrm{j} / \mathrm{mol} \cdot \mathrm{K}\right)$ indicate a spontaneous endothermic process [74].

The Reactive Yellow 145 dye adsorption process by crushed watermelon seeds pretreated with hexane in the Soxhlet apparatus was studied. From the Langmuir equation, it was found that the maximum sorption capacity of watermelon seeds for the above dye can be $115 \mathrm{mg} / \mathrm{g}$. Actually, it was slightly more than $83 \mathrm{mg} / \mathrm{g}$. It was found that the Freundlich equation 
more accurately describes adsorption isotherm, and the process kinetics follows the pseudosecond-order model [75].

Urban wastewater was treated using watermelon seeds as a coagulant and biofilter media. It was noted that the use of the latter allows reducing the values of BOD by $91 \%$, COD by $79 \%$, the content of nitrate ions by $26.3 \%$, and nitrite ions by $95 \%$ [76]. It is stated that the use of a coagulative composition consisting of $80 \%$ crushed watermelon seeds and $20 \%$ alum can remove the turbidity and color of river water by $100 \%$ [77].

\section{The Use of Melon Fruit Waste as Materials to Absorb Pollutants from Water Media}

There is literature about using biomass and by-products of melon (Cucumis melo), a Cucumis plant in the gourd family, a cucurbit, https://ru.wikipedia.org/wiki/\%D0\%9E\%D0\%B3\%D1\%83\%D1\%80\%D0\%B5\%D1\%86_(\% $\mathrm{D} 1 \% 80 \% \mathrm{D} 0 \% \mathrm{BE} \% \mathrm{D} 0 \% \mathrm{~B} 4)$ as a sorption material. It is an annual herbaceous plant with creeping rounded-faceted stems with tendrils. Leaves are large, alternate, without stipules, round-ovate or palmate-lobed, on long petioles. Flowers are bisexual, pale yellow. The fruit is a pumpkin of a spherical or cylindrical shape with a strong leathery exocarp, juicy mesocarp, and endocarp; it may be of various colors and shapes, with white or greenish flesh [78].

The by-products of melon fruits processing are rind and seeds, which can be used to produce various biologically active agents, as a substrate for solid-phase fermentation, etc. $[19,79]$.

Besides, the dried melon rind was studied as a sorption material to remove pollutants from aqueous media. In particular, the melon rind was used to remove $\mathrm{Cd}^{2+}$ ions from the water phase. The results indicate an increase in the absorption of $\mathrm{Cd}^{2+}$ ions at an increase in the initial concentration of $\mathrm{Cd}^{2+}$ ions, the solution $\mathrm{pH}$, and the contact time. The maximum sorption capacity was found to be $81.97 \mathrm{mg} / \mathrm{g}$. It was found that the Langmuir model provides the best correlation of the experimental data, and the pseudo-second-order model is the most applicable for describing the kinetics of $\mathrm{Cd}^{2+}$ ion adsorption by melon rind [80].

$\mathrm{Cu}^{2+}$ ions were removed in the dynamic mode of adsorption using dried melon rind as the sorption material. Initial concentrations of $\mathrm{Cu}^{2+}$ ions ranged from 20 to $200 \mathrm{mg} / \mathrm{dm}^{3}$, and the height of the sorption material layer was $0.5-1.25 \mathrm{~m}$. It was found that an increase in the melon rind layer and a decrease in $\mathrm{Cu}^{2+}$ ion concentration resulted in increased channeling time [81].

The dried melon rind was used to remove $\mathrm{Fe}^{2+}, \mathrm{Mn}^{2+}$, and $\mathrm{Pb}^{2+}$ ions from groundwater with varying parameters: $\mathrm{pH}$ value, biosorbent dosage, initial metal ion concentration contact time. It was found that adsorption was most effective at $\mathrm{pH}=7$ for $\mathrm{Fe}^{2+}$ ions and $\mathrm{pH}=6.5$ for $\mathrm{Mn}^{2+}$ and $\mathrm{Pb}^{2+}$ ions at a biosorbent dosage of $0.5 \mathrm{~g} / \mathrm{dm}^{3}$ and a contact time of 45 minutes. Under these conditions, it was found that up to $90.73 \%, 91.47 \%$, and $90.94 \%$ of the named ions are removed, respectively. It was found that the Langmuir model more accurately describes the adsorption isotherms, and the kinetics of the process corresponds to the pseudo-second-order model. The maximum adsorption capacity found from the Langmuir equation was $5.35 \mathrm{mg} / \mathrm{g}$, $2.75 \mathrm{mg} / \mathrm{g}$, and $0.083 \mathrm{mg} / \mathrm{g}$ for $\mathrm{Fe}^{2+}, \mathrm{Mn}^{2+}$, and $\mathrm{Pb}^{2+}$ ions, respectively [82-84].

Melon rind was studied to remove the Methylene Blue dye under static conditions. The maximum sorption capacity was found to be $333 \mathrm{mg} / \mathrm{g}$ [91]. Experiments described in [86] determined that the Methylene Blue dye's maximum sorption capacity is $46.4 \mathrm{mg} / \mathrm{g}$. This value was determined at an initial dye concentration of $500 \mathrm{mg} / \mathrm{dm}^{3}$. It was determined that in both works, the adsorption isotherm is adequately described by the Langmuir model. The kinetics 
of the process corresponds to the pseudo-second-order model $[85,86]$. Experiments were also performed under dynamic conditions with varying initial dye concentrations and flow rates. It was found that high layer height, low flow rate, and high initial dye concentration are the best conditions for maximum dye adsorption. It was determined that graphic dependencies are well described by the models of Bohart-Adams, Clark, Yoon, and Nelson [87].

Melon processing by-products also include seeds. Experiments were performed on the adsorption of $\mathrm{Pb}^{2+}$ ions by crushed native Cucumis melo seeds. It was found that an increase in the dosage of the biosorbent provides an increase in the degree of pollutant removal. It was determined that the Langmuir model more accurately describes the adsorption isotherm with a maximum biosorption capacity of the monolayer of $3.64 \cdot 10^{-4} \mathrm{~mol} / \mathrm{g}$, and the kinetics of the process corresponds to the pseudo-second-order model. The calculated thermodynamic parameters indicate that $\mathrm{Pb}^{2+}$ ions biosorption on melon rind biomass is a spontaneous and endothermic process [88].

Melon seed husks have also been studied as a sorption material. In particular, it was shown that melon seed husks could be used to remove Methylene Blue from model solutions with an initial concentration of $30 \mathrm{mg} / \mathrm{dm}^{3}$. It was found that at $\mathrm{pH}=7$, the dosage of melon seed husk $1.5 \mathrm{~g} / \mathrm{dm}^{3}$ after 150 minutes of contact, the dye removal efficiency was $91.6 \%$. It was determined that the adsorption isotherm and the kinetic parameters of the process best corresponded to the Tyomkin isotherm and the pseudo-first-order kinetic model [89].

The world literature provides several publications on the use of muskmelon components, aka cantaloupe (Cucumis melo var. cantalupensis), a plant in the gourd family (Cucurbitaceae), a melon type. Cantaloupe fruits are covered with a striped rind. The length of the fruit is $15-25 \mathrm{~cm}$. Its tender orange pulp is juicy, dense, sweet, and fragrant. Inside, there is a voluminous seed cavity with many oval flat grains [90].

Native dried and polyvinyl alcohol-modified cantaloupe rind was studied, in particular, for the adsorption of $\mathrm{Cd}^{2+}$ ions with an initial concentration of $50 \mathrm{mg} / \mathrm{dm}^{3}$ from simulated solutions. It was found that the efficiency of removing these ions was 71 and 79\%, respectively, with $15 \mathrm{~g} / \mathrm{dm}^{3}$ sorption materials and 100 minutes contact time. It was found that the Langmuir model well describes the adsorption isotherm, and the kinetics of the process is well described by the pseudo-second-order model. Based on the process's calculated thermodynamic parameters, it was determined that the adsorption process is endothermic and spontaneous [91].

Cantaloupe rind modified with $\mathrm{Ca}(\mathrm{OH})_{2}$ suspension was also studied for the ability to remove $\mathrm{Pb}^{2+}$ ions from simulated solutions. It was found that the maximum adsorption capacity for $\mathrm{Pb}^{2+}$ ions was $0.81 \mathrm{~mol} / \mathrm{kg}$ at an initial concentration of $1 \mathrm{~mol} / \mathrm{dm}^{3}$. As in the previous case, adsorption isotherm is well described by the Langmuir model, and the kinetics equation is well described by the pseudo-second-order model [92].

Cantaloupe seed husks were used to remove the butachlor pesticide from aqueous solutions. It was determined that the highest sorption capacity for butachlor was $142.8 \mathrm{mg} / \mathrm{kg}$ at $\mathrm{pH}=3$. The adsorption isotherm is well described by the Freundlich model $\left(\mathrm{R}^{2}=0.9977\right)$, and the pseudo-second-order model describes the process kinetics. Based on the calculated thermodynamic parameters of the process $\left(\Delta \mathrm{G}^{\mathrm{o}}=-0.646--3.989 \mathrm{~kJ} / \mathrm{mol}(308-343 \mathrm{~K}), \Delta \mathrm{H}^{\circ}-\right.$ $\left.20.91 \mathrm{~J} / \mathrm{mol}, \Delta \mathrm{S}^{\mathrm{o}}=-68.0 \mathrm{~J} / \mathrm{mol} \cdot \mathrm{K}\right)$, the adsorption process is exothermic and spontaneous [93]. 


\section{The Use of Cucumber Rind as Sorption Materials for Removal of Metal Ions and Dyes from Aqueous Media}

The gourd family includes cucumbers (Cucurbita), which are widely distributed around the globe. Cucumber or ridge cucumber (Cucumis sativus) is an annual herbaceous plant, a Cucumber (Cucumis) species in the gourd family (Cucurbitaceae), a vegetable crop. The stem is creeping, rough ends in tendrils, which it can catch on the support, stretching out for 1-2 m. Leaves are heart-shaped, five-lobed. The fruit is multi-seeded, juicy, emerald-green, pimply. The fruit's structure is characteristic of the gourd family and is defined in Botanics as a pumpkin. It can be in different shapes and sizes (depending on the variety) [94].

One hundred nine compounds were found in the rind, seeds, and pulp of cucumbers. Research has shown that cucumber rind and seeds contain rich nutrients, such as aromatic compounds, $\beta$-tocopherol, squalene, zingiberene, cathine, linoleic acid, and others [95]. Such saccharides as rhamnose, arabinose, xylose, mannose, and glucose were also found [96].

Biomass and by-products from cucumber processing have also been studied as sorption materials for removing pollutants from aquatic environments. For example, a dried rind of cucumbers was studied to remove $\mathrm{Cd}^{2+}$ ions from water media under static and dynamic conditions [97-100]. It was determined that the maximum sorption capacity for $\mathrm{Cd}^{2+}$ ions is $0.998 \mathrm{mmol} / \mathrm{g}$ [97], and adsorption proceeds for functional - $\mathrm{OH}$ groups that are part of biopolymers and other compounds.

In [98], Cd2+ ions adsorption by cucumber rind under static conditions was also studied. It was found that at an initial concentration of $10 \mathrm{mg} / \mathrm{dm}^{3}, \mathrm{pH}=5$, temperature $25{ }^{\circ} \mathrm{C}$, and sorption material dosage of $2 \mathrm{~g} / \mathrm{dm}^{3}$, the maximum efficiency of $\mathrm{Cd}^{2+}$ ions removal was $90.2 \%$. It was found that the Freundlich model more accurately describes the adsorption isotherm, and the kinetics of the process corresponds to the pseudo-second-order model. The calculated thermodynamic parameters of the process revealed that the adsorption process is exothermic and spontaneous.

$\mathrm{Cd}^{2+}$ ions removal by crushed cucumber rind under dynamic conditions was also studied. It was determined that at the initial concentration of cadmium ions of $50 \mathrm{mg} / \mathrm{dm}^{3}$, the liquid flow rate through an $8 \mathrm{~cm}$ layer of sorption material was $20 \mathrm{~cm}^{3} / \mathrm{min}$, the efficiency of pollutant removal was $78.03 \%$, with the maximum sorption capacity of $107.76 \mathrm{mg} / \mathrm{g}$ [99].

To increase cucumber rind adsorption capacity for $\mathrm{Cd}^{2+}$ ions, the rind was treated with $\mathrm{HCl}$ solution. At an initial $\mathrm{Cd}^{2+}$ ions concentration of $20 \mathrm{mg} / \mathrm{dm}^{3}$, the maximum adsorption capacity increased from $3.6 \mathrm{mg} / \mathrm{g}$ [98] to $58.1 \mathrm{mg} / \mathrm{g}$ at $298 \mathrm{~K}$ [100]. It was found that the adsorption isotherm, in this case, is more accurately described by the Langmuir model with the same process parameters.

The work also included studying $\mathrm{Pb}^{2+}$ ions adsorption by dried cucumber rind under static conditions. It was found that the maximum adsorption capacity for lead ions is $28.25 \mathrm{mg} / \mathrm{g}$ at the initial concentration of the latter in a solution of $25 \mathrm{mg} / \mathrm{dm}^{3}$ and a temperature of $30^{\circ} \mathrm{C}$. It was found that the adsorption isotherm is most accurately described by the Langmuir model and the kinetics of the process by the pseudo-second-order model. It was determined that adsorption is spontaneous and exothermic [101]. At the same time, [102] states that the maximum adsorption capacity of cucumber rind for $\mathrm{Pb}^{2+}$ ions is $133.6 \mathrm{mg} / \mathrm{g}$ at a temperature of $30^{\circ} \mathrm{C}, \mathrm{pH}=5$, and a contact time of 60 minutes.

Cucumber rind has also been studied as a sorption material for removing $\mathrm{Cu}^{2+}$ and $\mathrm{Pb}^{2+}$ ions [103]. It was determined that the maximum adsorption capacity for these ions is 88.5 and 
$147.1 \mathrm{mg} / \mathrm{g}$, respectively. These parameters were achieved at an initial ion concentration of $100 \mathrm{mg} / \mathrm{dm}^{3}\left(\mathrm{Cu}^{2+}\right)$ and $150 \mathrm{mg} / \mathrm{dm}^{3}\left(\mathrm{~Pb}^{2+}\right), \mathrm{pH}=5$, and a contact time of 60 and 85 minutes, respectively.

Dried cucumber rind has also been studied to extract various dyes from aqueous media [104-109]. In particular, it was determined that the maximum adsorption capacity of cucumber rind for the Crystal Violet dye is $149.25 \mathrm{mg} / \mathrm{g}$; the Langmuir model describes the adsorption isotherm, and the kinetics of the process corresponds to the pseudo-second-order model [105]. In [106], it was determined from the Langmuir equation that the maximum sorption capacity of cucumber rind for the above dye is $34.24 \mathrm{mg} / \mathrm{g}$. At the initial Crystal Violet concentration of $25 \mathrm{mg} / \mathrm{dm}^{3}, \mathrm{pH}=7$, and the rind dosage of $4 \mathrm{~g} / \mathrm{dm}^{3}$, the degree of dye removal was $92.15 \%$.

The study also involved the removal of the Acid Red 1 dye by dried cucumber rind. With the initial dye concentration of $100 \mathrm{mg} / \mathrm{dm}^{3}$, the rind dosage of $12.5 \mathrm{~g} / \mathrm{dm}^{3}$ and $\mathrm{pH}=2$, the maximum sorption capacity was $3.21 \mathrm{mg} / \mathrm{g}$ [107]. It was determined that the adsorption isotherm is more accurately described $\left(\mathrm{R}^{2}=0.984\right)$ by the Dubinin-Radushkevich model.

Methylene blue and Orange G's sorption capacity was determined and compared of banana, cucumber, and tomato skins. It was found that the maximum adsorption capacity of cucumber rind for Methylene blue was $179.9 \mathrm{mg} / \mathrm{g}$, which is lower than that of banana skin and higher than that of tomato skin. For Orange $\mathrm{G}$, this indicator is $40.5 \mathrm{mg} / \mathrm{g}$ and exceeds the values of other sorption materials studied [108].

Crushed and dried cucumber rind was studied as a sorption material for removing Crystal Violet and Rhodamine B dyes. It was determined that the maximum sorption capacity for these dyes, determined by the Langmuir model equation, was $33.22 \mathrm{mg} / \mathrm{g}$ and $40.82 \mathrm{mg} / \mathrm{g}$, respectively. It was found that the adsorption isotherms are most accurately described by the Langmuir model and the kinetics of the process by the pseudo-second-order model [109].

Information about the use of marrows and scallop squashes biomass and by-products as sorption materials for removing pollutants from water media is not found in the literature.

\section{Conclusions}

As can be seen from the above, we analyzed and summarized the information on using cucurbits by-products. It was determined that dried rind of pumpkins, watermelons, melons, and cucumbers have good sorption properties concerning heavy metal ions and various dyes. Most of the adsorption isotherms are most accurately described by the Langmuir model, less often by the Freundlich model. It was determined that the adsorption process kinetics most often follows the pseudo-second-order model. To improve the adsorption characteristics for various pollutants, adsorption materials were modified with various chemicals.

\section{Funding}

This research received no external funding.

\section{Acknowledgments}

This research has no acknowledgment.

\section{Conflicts of Interest}

The authors declare no conflict of interest. 


\section{References}

1. Chakraborty, R.; Asthana, A.; Singh, A.K.; Jain, B.; Susan, A.B.H. Adsorption of heavy metal ions by various low-cost adsorbents: a review. Int. J. Environ. Analyt. Chem. 2020, 1-38, https://doi.org/10.1080/03067319.2020.1722811.

2. Shaikhiev, I.G.; Sverguzova, S.V.; Galimova, R.Z.; Grechina, A.S. Using wastes of buckwheat processing as sorption materials for the removal of pollutants from aqueous media: a review. IOP Conf. Series: Materials Science and Engineering. 2020, 945, https://doi.org/10.1088/1757-899X/945/1/012044.

3. Chikri, R.; Elhadiri, N.; Benchanaa, M.; El maguana, Y. Efficiency of sawdust as low-cost adsorbent for dyes removal. J. Chem. 2020, 2020, 1-17, https://doi.org/10.1155/2020/8813420.

4. Crini, G.; Lichtfouse, E.; Wilson, L.D.; Crin, N.M. Conventional and nonconventional adsorbents for wastewater treatment. Environ. Chem. Let. 2019, 17, 195-213, https://doi.org/10.1007/s10311-018-0786-8.

5. Yeow, P.K.; Wong, S.W; Hadibarata, T. Removal of Azo and Anthraquinone Dye by Plant Biomass as Adsorbent - A Review. Biointerface Res. ApChem. 2021, 11, 8218-8232, https://doi.org/10.33263/BRIAC111.82188232.

6. Varghese, A.G.; Paul, S.A.; Latha, M.S. Remediation of heavy metals and dyes from wastewater using cellulose based adsorbents. Environ. Chem. Let. 2019, 17, 867-877, https://doi.org/10.1007/s10311-01800843-z.

7. Tsade, H.; Murthy, H.C.A.; Muniswamy, D. Bio-sorbents from agricultural wastes for eradication of heavy metals: A review. J. Mater. Environ. Sci. 2020, 11, 1719-1735.

8. Panigrahi, T.; Santhoskumar, A.U. Adsorption process for reducing heavy metals in textile industrial effluent with low cost adsorbents. Prog. Chem. Biochem. Res. 2020, 3, 135-139, https://doi.org/10.33945/SAMI/PCBR.2020.2.7.

9. Zhou, Y.; Lu, J.; Zhou, Y.; Liu, Y. Recent advances for dyes removal using novel adsorbents: A review. Environ. Pollut. 2019, 252, 352-365, https://doi.org/10.1016/j.envpol.2019.05.072.

10. Sverguzova, S.V., Shaikhiev, I.G., Fomina, E.V.; Galimova, R.Z. Use of chestnut sheel (Castánea) as adsorption material for removing pollutants from natural and sewage waters: a review. IOP Conf. Series: Materials Science and Engineering. 2020, 945, https://doi.org/10.1088/1757-899X/945/1/012072.

11. Ngwenya, S.; Guyo, U.; Zinyama, N.P.; Chigondo, F.; Nyamunda, B.C.; Muchanyereyi, N. Response surface methodology for optimization of Cd(II) adsorption from wastewaters by fabricated tartaric acid-maize tassel magnetic hybrid sorbent. Biointerface Res. ApChem. 2019, 9, 3996-4005, https://doi.org/10.33263/BRIAC94.996005.

12. Chong, S.N.; Hadibarata, T. Adsorption of phenol red and remazol brilliant blue R by coconut shells (Cocos nucifera) and ambarella peels (Spondias dulcis). Biointerface Res. ApChem. 2021, 11, 8564-8576, https://doi.org/10.33263/BRIAC111.85648576.

13. Shaikh, T.M.A. Adsorption of $\mathrm{Pb}$ (II) from wastewater by natural and synthetic adsorbents. Biointerface Res. ApChem. 2020, 5, 6522-6539, https://doi.org/10.33263/BRIAC105.65226539.

14. Kanthasamy, S.; Hadibarat, T.; Hidayat, T.; Alamri, S.A.; Al-Ghamdi, A.A. adsorption of azo and anthraquinone dye by using watermelon peel powder and corn peel powder: equilibrium and kinetic studies. Biointerface Res. Appl. Chem. 2020, 10, 4706-4713, https://doi.org/10.33263/BRIAC101.706713.

15. Robinson, R.W.; Decker-Walters, D.S. Cucurbits. CAB International. Wallingford, Oxford, UK, $1999 ;$ pp. 226.

16. Sánchez-Hernández, M.A.; Sánchez-Hernández, C.; Villanueva-Verduzco, C.; Gil-Vázquez, I.; JiménezRojas, M.C.; Sánchez-Cabrera, I. Multiplicacion in vitro via organogenesis en calabaza. Agronomía Mesoamer. 2009, 20, 11-22.

17. Habib, A.; Biswas, S.; Siddique, A.H.; Manirujjaman, M.; Uddin, B.; Hasan, S.; Khan, M.M.H.; Uddin, M.; Islam, M.; Hasan, M.; Rahman, M.; Asaduzzaman, M.; Rahman, M.S.; Khatun, M.; Islam, M.A.; Rahman, M. Nutritional and lipid composition analysis of pumpkin seed (Cucurbita maxima Linn.). J. Nutrit. Food Sci. 2015, 5, 1-6, https://doi.org/10.4172/2155-9600.1000374.

18. Kipping, D.R.; Laurel, H.O.; Orozco, A.A.; García, H.M.D.; Martínez, L.A.L. Características físicas y químicas de la semilla de calabaza para mecanización y procesamiento. Nova Scientia. 2018, 10, 61-77, https://doi.org/10.21640/ns.v10i21.1467.

19. Shetty, A.A.; Rana, R.; Buckseth, T.; Preetham, S.P. Waste utilization in cucurbits: A review. Waste Biomass Valor. 2012, 3, 363-368, https://doi.org/10.1007/s12649-012-9114-X.

20. Çelekli, A.; Bozkuş, B.; Bozkurt, H. Development of a new adsorbent from pumpkin husk by KOHmodification to remove copper ions. Environ. Sci. Pollut. Res. 2019, 26, 11514-11523, https://doi.org/10.1007/s11356-017-1160-2.

21. Eze, S.O.; Igwe, J.C.; Dipo, D. Effect of particle size on adsorption of heavy metals using chemically modified and unmodified fluted pumpkin and broad-leafed pumpkin pods. Int. J. Biolog. Chem. Sci. 2013, 7, 852-860, https://doi.org/10.4314/ijbcs.v7i2.40.

22. Çelekli, A.; Bozkurt, H. Predictive modeling of an azo metal complex dye sorption by pumpkin husk. Environ. Sci. Pollut. Res. 2013, 20, 7355-7366, https://doi.org/10.1007/s11356-013-1751-5. 
23. Çelekli, A.; Çelekli, F.; Çiçek, E.; Bozkurt, H. Predictive modeling of sorption and desorption of a reactive azo dye by pumpkin husk. Environ. Sci. Pollut. Res. 2014, 21, 5086-5097, https://doi.org/10.1007/s11356013-2452-9.

24. Çelekli, A.; Küçükgüner, B.; Bozkurt, H. Diazo dye sorption by Ni-modified pumpkin husk. Desalin. Water Treat. 2016, 57, 25242-25255, https://doi.org/10.1080/19443994.2016.1149740.

25. Swelam, A.A.; Awad, M.B.; Salem, A.M.A. Kinetics study on the removal of Cu(II) from aqueous solution using raw and modified pumpkin seed hulls - low cost biosorbents. Int. J. Environ. 2015, 4, 38-50.

26. Hameed, B.H.; El-Khaiary, M.I. Removal of basic dye from aqueous medium using a novel agricultural waste material: Pumpkin seed hull. J. Hazard. Mater. 2008, 155, 601-609, https://doi.org/10.1016/j.jhazmat.2007.11.102.

27. Celebi, H. The applicability of evaluable wastes for the adsorption of Reactive Black 5. Int. J. Environ. Sci. Technol. 2019, 16, 135-146, https://doi.org/10.1007/s13762-018-1969-3.

28. Apostol, L.C.; Ghinea, C.; Alves, M.; Gavrilescu, M. Removal of erythrosine B dye from water effluents using crop waste pumpkin seed hulls as adsorbent. Desalinat. Water Treat. 2016, 57, 22585-22608, https://doi.org/10.1080/19443994.2015.1132477.

29. Subbaiah, M.V.; Kim, D. Adsorption of methyl orange from aqueous solution by aminated pumpkin seed powder: kinetics, isotherms, and thermodynamic studies. Ecotoxicol. Environ. Saf. 2016, 128, 109-117, https://doi.org/10.1016/j.ecoenv.2016.02.016.

30. Kusmierek, K.; Swiatkowski, A.; Dabek, L. Removal of 2,4,6-trichlorophenol from aqueous solutions using agricultural waste as low-cost adsorbents. Environ. Protec. Eng. 2017, 43, 149-163..

31. Menkiti, M.; Chime, T.; Onukwuli, O. Bioadsorption of suspended and dissolved particles from coal washery effluent onto fluted pumpkin seed shell biomass. World J. Eng. 2011, 8, 179-190.

32. Eseyin, O.A.; Sattar, M.A.; Rathore, H.A. A review of the pharmacological and biological activities of the aerial parts of Telfairia occidentalis Hook.f. (Cucurbitaceae). Trop. J. Pharm. Res. 2014, 13, 1761-1769.

33. Horsfall, Jr.M.; Spiff, A.I. Sorption of lead, cadmium and zinc of sulfur-containing chemically modified wastes of fluted pumpkin (Telfairia occidentalis Hook f). Chem. Biodivers. 2005, 2, 373-385, https://doi.org/10.1002/cbdv.200590017.

34. Horsfall, J.M.; Spiff, A.I. Adsorption of transition metals in aqueous solutions by fluted pumpkin (Telfairia occidentalis Hook f). Chem. Biodivers. 2005, 2, 1266-1276, https://doi.org/10.1002/cbdv.200590097.

35. Horsfall, J.M.; Spiff, A.I. Equilibrium sorption study of $\mathrm{Al}^{3+}, \mathrm{Co}^{2+}$ and $\mathrm{Ag}^{+}$in aqueous solutions by fluted pumpkin (Telfairia occidentalis HooK f) waste biomass. Acta Chim. Slovenica. 2005, 52, 174-181.

36. Horsfall, Jr.M.; Spiff, A.I. Effect of 2-mercaptoethanoic acid treatment of fluted pumpkin waste (Telfairia occidentalis Hook f) on the sorption of $\mathrm{Ni}^{2+}$ ions from aqueous solution. J. Scie. Ind. Res. 2005, 64, 613-620.

37. Barajas, J.; Cano, M.; Castorena, J.H.; Santiago, V.; Díaz, J. Remocion de colorantes por medio de Curcubitaceas. Avances en Ciencias e Ingeniería. 2016, 7, 67-80.

38. Erhirhie, E.O.; Ekene, N.E. Medicinal values on Citrullus lanatus (Watermelon): pharmacological review. Int. J. Research Pharmac. Biomed. Sci. 2013, 4, 1305-1312.

39. Egbuonu, A.C.C. Comparative assessment of some vineral, amino acid and vitamin compositions of watermelon (Citrullus lanatus) rind and seed. Asian J. Biochem. 2015, 10, 230-236, http://dx.doi.org/10.3923/ajb.2015.230.236.

40. Shakoor, M.B.; Niazi, N.K.; Bibi, I.; Shahid, M.; Sharif, F.; Bashir, S.; Shaheen, S.M.; Wang, H.; Tsang, D.C.W.; Ok, Y.S.; Rinklebe, J. Arsenic removal by natural and chemically modified watermelon rind in aqueous solutions and groundwater. Sci. Total Environ. 2018, 645, 1444-1455, http://dx.doi.org/10.1016/j.scitotenv.2018.07.218.

41. Lakshmipathy, R.; Vinod, A.V.; Sarada, N.C. Watermelon rind as biosorbent for removal of $\mathrm{Cd}^{2+} \mathrm{from}$ aqueous solution: FTIR, EDX and kinetic studies. J. Indian Chem. Soc. 2013, 90, 1147-1154.

42. Reddy, N.A.; Lakshmipathy, R.; Sarada, N.C. Application of Citrullus lanatus rind as biosorbent for removal of trivalent chromium from aqueous solution. Alexandria Eng. J. 2014, 53, 969-975, http://dx.doi.org/10.1016/j.aej.2014.07.006.

43. Liu, C.; Ngo, H.H.; Guo, W.; Tung, K. Optimal conditions for preparation of banana peels, sugarcane bagasse and watermelon rind in removing copper from water. Biores. Technol. 2012, 119, 349-354, http://dx.doi.org/10.1016/j.biortech.2012.06.004.

44. Mohammed, T.J.; Ibrahim, R.I. Remediation of $\mathrm{Cu}(\mathrm{II})$ from well water of Iraq by using cortex of fruits and agricultural waste. Arabian J. Sci. Eng. 2016, 41, 345-355, http://dx.doi.org/10.1007/s13369-015-1848-x.

45. Banerjee, K.; Ramesh, S.T.; Gandhimathi, R.; Nidheesh, P.V.; Bharathi, K.S. A novel agricultural waste adsorbent, watermelon shell for the removal of copper from aqueous solutions. Iranica J. Energy Environ. 2012, 3, 143-156.

46. Gupta, H.; Gogate, P.R. Intensified removal of copper from waste water using activated watermelon based biosorbent in the presence of ultrasound. Ultrasonics Sonochem. 2016, 30, 113-122, http://dx.doi.org/10.1016/j.ultsonch.2015.11.016.

47. Mathangi, J.B.; Kalavathy, M.H. Study of mathematical models for the removal of $\mathrm{Ni}^{2+}$ from aqueous solutions using Citrullus lanatus rind, an agro-based waste. Water Environ. J. 2019, 33, 276-291, http://dx.doi.org/10.1111/wej.12408. 
48. Ibrahim, A.; Yusof, L.; Beddu, N.S.; Galasin, N.; Lee, P.Y.; Lee, R.N.S.; Zahrim, A.Y. Adsorption study of ammonia nitrogen by watermelon rind. IOP Conf. Series: Earth and Environ. Sci. 2016, 36, http://dx.doi.org/10.1088/1755-1315/36/1/012020.

49. Zahrim, A.Y.; Lija, Y.; Ricky, L.N.S.; Azreen, I. Fruit waste adsorbent for ammonia nitrogen removal from synthetic solution: Isotherms and kinetics. IOP Conf. Series: Earth Environ. Sci. 2016, 36, http://dx.doi.org/10.1088/1755-1315/36/1/012028.

50. Kiew, P.L.; Toong, J.F. Screening of significant parameters affecting Zn (II) adsorption by chemically treated watermelon rind. Progress Energy Environ. 2018, 6, 19-32.

51. Othman, N.; Kueh, Y.S.; Azizul-Rahman, F.H.; Hamdan, R. Watermelon rind: A potential adsorbent for zinc removal. App. Mechan. Mater. 2014, 680, 146-149, https://doi.org/10.4028/www.scientific.net/AMM.680.146.

52. Lakshmipathy, R.; Sarada, N.C. Application of watermelon rind as sorbent for removal of nickel and cobalt from aqueous solution. Inter. J. Miner. Proc. 2013, 122, 63-65, http://dx.doi.org/10.1016/j.minpro.2013.03.002.

53. Lakshmipathy, R.; Sarada, N.C. Metal ion free watermelon (Citrullus lanatus) rind as adsorbent for the removal of lead and copper ions from aqueous solution. Desalin. Water Treat. 2016, 57, 15362-15372, https://doi.org/10.1080/19443994.2015.1072064.

54. Othman, N.; Kadir, A.A.; Mohamed, R.M.S.; Azizul-Rahman, M.F.H.; Hamdan, R.; Chay, T.C. Characterization of watermelon rind as a biosorbent in removing zinc and lead. Int. conf. «Advances in Civil, Architectural, Structural and Constructional Engineering», South Korea, Busan 2015, 37-40.

55. Azizul-Rahman, M.F.H.; Mohd-Suhaimi, A.A.; Othman, N. Biosorption of $\mathrm{Pb}(\mathrm{II})$ and $\mathrm{Zn}(\mathrm{II})$ in synthetic waste water by watermelon rind (Citrullus lanatus). App. Mechan. Mater. 2014, 465-466, 906-910, http://dx.doi.org/10.4028/www.scientific.net/AMM.465-466.906.

56. Liu, C.; Ngo, H.H.; Guo, W. Watermelon rind: agro-waste or superior biosorbent? App. Biochem. Biotechnol. 2012, 167, 1699-1715, http://dx.doi.org/10.1007/s12010-011-9521-7.

57. Othman, N.; Azhar, N.; Rani, M.A.; Zaini, H.M. Metal removal and antimicrobial properties of watermelon rind modified with clove. MATEC Web Conf. 2016, 7, http://dx.doi.org/10.1051/matecconf/20167801028.

58. Jawad, A.H.; Ngoh, Y.S.; Radzun, K.A. Utilization of watermelon (Citrullus lanatus) rinds as a natural lowcost biosorbent for adsorption of methylene blue: kinetic, equilibrium and thermodynamic studies. J. Taibah Univ. Sci. 2018, 12, 371-381, http://dx.doi.org/10.1080/16583655.2018.1476206.

59. Lakshmipathy, R.; Sarada, N.C. Methylene blue adsorption onto native watermelon rind: batch and fixed bed column studies. Desalin. Water Treat. 2016, 57, 10632-10645, http://dx.doi.org/10.1080/19443994.2015.1040462.

60. Lakshmipathy, R.; Reddy, N.A.; Sarada, N.C. Optimization of brilliant green biosorption by native and acidactivated watermelon rind as low-cost adsorbent. Desalin. Water Treat. 2015, 54, 235-244, http://dx.doi.org/10.1080/19443994.2013.879082.

61. Ibrahim, M.B.; Sani, S. Comparative isotherms studies on adsorptive removal of Congo Red from wastewater by watermelon rinds and neem-tree leaves. Open J. Phys. Chem. 2014, 4, 139-146, http://dx.doi.org/10.4236/ojpc.2014.44017.

62. Sani, S.; Abdullahi, H. Kinetics and thermodynamics of methyl orange uptake from model effluent by watermelon shells and neem leaves. Int. J. Adv. Chem. Eng. Biol. Sci. 2017, 4, http://dx.doi.org/10.15242/IJACEBS.ER0117011.

63. Krueger, M.D. de S.; Volkmann, A.C.; Rainert, K.T. Removal of textile dye Remazol Brilliant Blue Reactive (RBBR) using fibers of Citrullus lanatus (watermelon) and Cocos nucifera (green coconut) as adsorbent material. Universid. Fed. de Santa Maria REGET, Santa Maria. 2019, 23, http://dx.doi.org/10.5902/2236117038526.

64. Rehman, R.; Mahmud, T. Sorptive elimination of Alizarin Red-S dye from water using Citrullus lanatus peels in environmentally benign way along with equilibrium data modeling. Asian J. Chem. 2013, 25, 53515356.

65. Rehman, R.; Shareef, A. Mechanistic and characterization studies for sorptive elimination of Emerald green dye from water by Arachis hypogeal shells and Citrullus lanatus peels. Asian J. Chem. 2014, 26, 2671-2676.

66. Ibrahim, M.B.; Ahmed, A. Adsorption of dyes onto activated watermelon (Citrullus lanatus) rinds (AWR). Bayero J. Pure App. Sci. 2017, 10, 606-614.

67. Chigbundu, E.C.; Adebowale, K.O. Equilibrium and fractal-like kinetic studies of the sorption of acid and basic dyes onto watermelon shell (Citrullus vulgaris). Cellulose 2017, 24, 4701-4714, http://dx.doi.org/10.1007/s10570-017-1488-2.

68. Latif, S.; Rehman, R.; Imran, M.; Iqbal, S.; Kanwal, A.; Mitu, L. Removal of acidic dyes from aqueous media using Citrullus lanatus peels: An agrowaste-based adsorbent for environmental safety. J. Chem. 2019, 2019, 1-9, http://dx.doi.org/10.1155/2019/6704953.

69. Lakshmipathy, R.; Sarada, N.C. Adsorptive removal of basic cationic dyes from aqueous solution by chemically protonated watermelon (Citrullus lanatus) rind biomass. Desalin. Water Treat. 2014, 52, 61756184, http://dx.doi.org/10.1080/19443994.2013.812526. 
70. Alexander, J.; Jayanthi, G.; Lakshmipathy, R.; Kulasekaran, A.; Andal, V. Colour removal studies on treatment of textile dyeing effluent by chitosan modified watermelon rind composite (CWR). Int. J. ChemTech Res. 2015, 8, 10-15.

71. Salman, J.M.; Al-Muttarii, A.K.; Abd-Hussain, N.A.; Jabour, A.M. Adsorption study of cadmium on water melon seed shell by flam atomic absorption spectroscopy (FAAS). Int. J. Sci. Eng. Res. 2015, 6, 832-836.

72. Akkaya, G.; Güzel, F. Bioremoval and recovery of $\mathrm{Cu}(\mathrm{II})$ and $\mathrm{Pb}$ (II) from aqueous solution by a novel biosorbent watermelon (Citrullus lanatus) seed hulls: Kinetic study, equilibrium isotherm, SEM and FTIR analysis. Desalin. Water Treat. 2013, 51, 7311-7322, http://dx.doi.org/10.1080/19443994.2013.815685.

73. Salman, J.M.; Abdal-Hussein, N.A.; Al-Muttairi, A.K. Removing the methylene blue dye from aqueous solutions by low cost materials. Int. J. Adv. Res. 2015, 3, 523-530.

74. Samra, S.E.; Jeragh, B.; EL-Nokrashy, A.M.; El-Asmy, A.A. Adsorption and thermodynamics of lead (II) using seeds of watermelon (SWM) as a low cost sorbent. Int. J. Eng. Appl. Sci. 2014, 1, 19-24.

75. Benkaddour, S.; Slimani, R.; Hiyane, H.; El Ouahabi, I.; Hachoumi, I.; El Antri, S.; Lazar, S. Removal of reactive yellow 145 by adsorption onto treated watermelon seeds: Kinetic and isotherm studies. Sustain. Chem. Pharm. 2018, 10, 16-21, http://dx.doi.org/10.1016/j.scp.2018.08.003.

76. Manyuchi, M.M.; Chikomo, T. Treatment of water using watermelon (Citrullus lanatus) seeds as organic coagulant and microbial filter. Int. Conf. Pure App. Chem., ICPAC 2016, Emerging Trends in Chemical Sciences. Flic en Flac, Mauritius 2016, 1-6.

77. Muhammad, I.M.; Abdulsalam, S.; Abdulkarim, A.; Bello, A.A. Water melon seed as a potential coagulant for water treatment. Global J. Res. Eng.: C Chem. Eng. 2015, 15, 1-9.

78. 78. McCreight, J.D.; Nerson, H.; Grumet R. Melon: Cucumis melo L. Genetic Improv. Veget. Crops, 1993, 267-294, https://doi.org/10.1016/B978-0-08-040826-2.50024-2.

79. Rolima, P.M.; Seabra, L.M.J.; de Macedo, G.R. Melon by-products: biopotential in human health and food processing. Food Rev. Int. 2019, 1-24, https://doi.org/10.1080/87559129.2019.1613662.

80. Hamdaoui, O.; Saoudi, F.; Chiha, M. Utilization of an agricultural waste material, melon (Cucumis melo L.) peel, as a sorbent for the removal of cadmium from aqueous phase. Desalin. Water Treat. 2010, 21, 228237, https://doi.org/10.5004/dwt.2010.1508.

81. Nieva, A.D.; Andres, J.C.S.; Gonzales, K.P. Simulated biosorption of $\mathrm{Cu}^{2+}$ in aqueous solutions using Cucumis melo VAR. cantalupensis. IOP Conf. Series: Earth and Environ. Sci. 2018, 191, https://doi.org/10.1088/1755-1315/191/1/012035.

82. Othman, N.; Asharuddin, S.M. Cucumis melo rind as biosorbent to remove Fe (II) and Mn (II) from synthetic groundwater solution. Adv. Mater. Res. 2013, 795, 266-271, https://doi.org/10.4028/www.scientific.net/AMR.795.266.

83. Othman, N.; Asharuddin, S.M. Utilization of Cucumis Melo rind as Fe and Pb ions biosorbent. In: 2nd IWA Malaysia Young Water Profes. Conf. 2015. Kuala Lumpur, Malaysia, 2015; pp. 1-14.

84. Asharuddin, S.B.M. Optimization of biosorption process using Cucumis melo rind for the removal of Fe, Mn and $\mathrm{Pb}$ ions from groundwater. A thesis submitted in fulfillment of the requirement for the award of the Degree of Master of Civil Engineering. University Tun Hussein Onn, Malaysia, 2015; pp. 92.

85. Djelloul, C.; Hamdaoui, O. Removal of cationic dye from aqueous solution using melon peel as nonconventional low-cost sorbent. Desalinat. Water Treat. 2014, 52, 7701-7710, https://doi.org/10.1080/19443994.2013.833555.

86. Olajire, A.A.; Giwa, A.A.; Bello, I.A. Adsorptive removal of methylene blue dye by melon husk: kinetic and isothermal studies. Pakistan J. Sci. Ind. Res. Series A; Phys. Sci. 2013; 56, 151-164.

87. Djelloul, C.; Hamdaoui, O. Dynamic adsorption of methylene blue by melon peel in fixed-bed columns. Desalinat. Water Treat. 2015, 56, 2966-2975, https://doi.org/10.1080/19443994.2014.963158.

88. Akar, S.T.; Arslan, S.; Alp, T.; Arslan, D.; Akar, T. Biosorption potential of the waste biomaterial obtained from Cucumis melo for the removal of $\mathrm{Pb}^{2+}$ ions from aqueous media: Equilibrium, kinetic, thermodynamic and mechanism analysis. Chem. Eng. J. 2012, 185-186, 82-90, https://doi.org/10.1016/j.cej.2012.01.032.

89. Rahimdokht, M.; Pajootan, E.; Arami, M. Application of melon seed shell as a natural low-cost adsorbent for the removal of Methylene Blue from dye-bearing wastewaters: optimization, isotherm, kinetic, and thermodynamic. Desalin. Water Treat. 2016, 57, 18049-18061, https://doi.org/10.1080/19443994.2015.1086698.

90. Bates, D.M.; Robinson, R.W. Cucumbers, melons and water melons. Cucumis and Citrullus (Cucurbitaceae). In: Evolution of crop plants. ed. by Smartt, J.; Simmonds, N.W.). 1995; pp. 89-96.

91. Chellam, A.G.; Shakina, J.; Usha, R. Studies of kinetics and thermodynamic parameters in the adsorption of cadmium (II) on Cucumis melo peel. Indian J. Sci. 2014, 9, 35-41.

92. Huang, K.; Zhu, H. Removal of $\mathrm{Pb}^{2+}$ from aqueous solution by adsorption on chemically modified muskmelon peel. Environ. Sci. Pollut. Res. 2013, 20, 4424-4434, https://doi.org/10.1007/s11356-012-13617.

93. Haq, A.; Saeed, M.; Usman, M.; Muneer, M.; Adeel, S.; Abbas, S.; Iqbal, A. Removal of butachlor from aqueous solution using cantaloupe seed shell powder: kinetic, equilibrium and thermodynamic studies. Int. J. Environ. Sci. Technol. 2018, 16, 6029-6042, https://doi.org/10.1007/s13762-018-1992-4. 
94. Tatlioglu, T. Cucumber: Cucumis sativus L. Genetic Improv. Veget. Crops. 1993, 197-234, https://doi.org/10.1016/B978-0-08-040826-2.50017-5.

95. Hao-xi, B.; Da-dong, L.; Ning-sheng, B.; Mei-fang, W. Studies on the chemical constituents of cucumber. Nat. Prod. Res. Develop. 2008, 20, 388-394.

96. Gross, K.C.; Wang, C.Y. Compositional changes in cell wall polysaccharides from chilled and non-chilled cucumber fruit. Phyfochem. 1984, 23, 1575-1578, ttps://doi.org/10.1016/S0031-9422(00)83442-1.

97. Basu, M.; Guha, A.K.; Ray, L. Adsorption of cadmium on cucumber peel: kinetics, isotherm and co-ion effect. Indian Chem. Eng. 2018, 60, 179-195, https://doi.org/10.1080/00194506.2017.1341349.

98. Pandey, R.; Ansari, N.G.; Murthy, R.C.; Prasad, R.L. Cd(II) adsorption from aqueous solution onto Cucumis sativus peel: equilibrium, thermodynamic and kinetic study. J. Ecophysiol. Occupat. Health. 2013, 3-4, 7584.

99. Basu, M.; Guha, A.K.; Ray, L. Adsorption of cadmium ions by cucumber peel in continuous mode. Int. J. Environ. Sci. Technol. 2019, 16, 237-248, https://doi.org/10.1007/s13762-017-1609-3.

100. Pandey, R.; Ansari, N.G.; Prasad, R.L.; Murthy, R.C. Removal of Cd(II) ions from simulated wastewater by $\mathrm{HCl}$ modified Cucumis sativus peel: equilibrium and kinetic study. Air, Soil, Water Res. 2014, 7, 93-101; https://doi.org/10.4137/ASWR.S16488.

101. Pandey, R.; Ansari, N.G.; Prasad, R.L.; Murthy, R.C. Cd(II) adsorption from aqueous solution onto Cucumis sativus peel: kinetic, equilibrium and thermodynamic study. Amer. J. Environ. Protect. 2014, 2, 51-58, https://doi.org/10.12691/env-2-3-1.

102. Basu, M.; Guha, A.K., Ray, L. Adsorption of lead on cucumber peel. J. Clean. Product. 2017, 151, 603615, https://doi.org/10.1016/j.jclepro.2017.03.028.

103. Akkaya, G.; Güzel, F. Optimization of copper and lead removal by a novel biosorbent: cucumber (Cucumis sativus) peels - kinetic, equilibrium, and desorption studies. J. Dispersion Sci. Nechnol. 2013, 34, https://doi.org/10.1080/01932691.2012.743863.

104. Shakoor, S.; Nasar, A. Adsorptive treatment of hazardous methylene blue dye from artificially contaminated water using Cucumis sativus peel waste as a low-cost adsorbent. Groundwater Sustain. Develop. 2017, 5, 152-159, https://doi.org/10.1016/j.gsd.2017.06.005.

105. Shakoor, S.; Nasar, A. Utilization of Cucumis sativus peel as an eco-friendly biosorbent for the confiscation of Crystal violet dye from artificially contaminated wastewater. Analyt. Chem. Lett. 2019, 9, 1-19, https://doi.org/10.1080/22297928.2019.1588162.

106. Smith, T.; Thirumalisamy, S.; Manonmani, S. Equilibrium and kinetics study of adsorption of Crystal violet onto the peel of Cucumis sativa fruit from aqueous solution. E-Journal Chem. 2012, 9, 1091-1101, https://doi.org/10.1155/2012/457632.

107. Khanna, S.; Rattan, V.K. Removal of Acid Red 1 from aqueous waste stream using peel of Cucumis Sativus fruit. Equilibrium studies. J. Chem. Technol. Metallurgy 2017, 52, 803-811.

108. Stavrinou, A.; Aggelopoulos, C.A.; Tsakiroglou, C.D. Exploring the adsorption mechanisms of cationic and anionic dyes onto agricultural waste peels of banana, cucumber and potato: Adsorption kinetics and equilibrium isotherms as a tool. J. Environ. Chem. Eng. 2018, 6, 6958-6970, https://doi.org/10.1016/j.jece.2018.10.063.

109. Smith, T.; Santhi, T.; Prasad, A.L.; Manonmani, S. Cucumis sativus used as adsorbent for the removal of dyes from aqueous solution. Arab. J. Chem. 2017, 10, S244-S251, https://doi.org/10.1016/j.arabjc.2012.07.030. 